\title{
Remarkable Histopathological Improvement of Experimental Toxoplasmosis Treated with Spiramycin-loaded Chitosan Nanoparticles
}

\section{Amal Farahat Allam ( $\square$ amalalam2005@yahoo.com ) \\ Alexandria University https://orcid.org/0000-0002-4965-9469}

\section{Nancy Hagras}

Pharos University in Alexandria

\section{Hoda Farag}

Alexandria University

\section{Mervat Osman}

Alexandria University

\section{Thanaa Shalaby}

Alexandria University

Amani Kazem

Alexandria University

Amel Shehab

Alexandria University

Nermine Hussein Mogahed

Alexandria University

\section{Original Article}

Keywords: Toxoplasma gondii, spiramycin, spiramycin-metronidazole, chitosan nanoparticles, spiramycin-chitosan nanoparticles formulation

Posted Date: February 3rd, 2021

DOl: https://doi.org/10.21203/rs.3.rs-160501/v1

License: (c) (i) This work is licensed under a Creative Commons Attribution 4.0 International License. Read Full License

Version of Record: A version of this preprint was published at Journal of Parasitic Diseases on August 10th, 2021. See the published version at https://doi.org/10.1007/s12639-021-01431-9. 


\section{Abstract}

The present study investigated the anti-Toxoplasma effect of chitosan nanoparticles [CS NPs], spiramycin, spiramycin co-administered with metronidazole and spiramycin-CS NPs formulation on the parasite burden and histopathological changes in the liver, spleen and brain in experimentally infected mice. Seventy male Swiss albino mice were classified into seven equal groups: healthy control (I), infected untreated control (II), infected group receiving CS NPs (III), spiramycin administered infected group (IV), infected group receiving spiramycin-metronidazole (V), infected receiving $400 \mathrm{mg} / \mathrm{kg}$ spiramycin-CS NPs (VI) and infected treated with spiramycin-loaded CS NPs $100 \mathrm{mg} / \mathrm{kg}$ (VII). All groups were inoculated intraperitoneally with $2500 \mathrm{~T}$. gondii tachyzoites $\mathrm{RH}$ strain except the healthy control group. All groups were sacrificed on the 8th day after infection. Density of the parasite and histopathological examination of the liver, spleen and brain of all treated mice revealed reduction in the mean tachyzoites count as well as decreased inflammation, congestion and necrosis within tissue sections. Spiramycin-loaded NPs displayed the highest significant reduction in the pathological insult tailed by spiramycin-metronidazole and CS NPs. In conclusion, spiramycin-loaded CS NPs showed a promising synergistic combination in the treatment of the histopathology caused by toxoplasmosis.

\section{Introduction}

Toxoplasmosis is a highly prevalent foodborne disease relating to the intracellular parasite Toxoplasma gondii [T. gondi]. It infects about one third of the population worldwide [Wohlfer et al. 2017]. The parasite exists in two distinct forms; a rapidly replicating invasive tachyzoite and a slowly replicating bradyzoite inside tissue cysts. T. gondii [RH strain] tachyzoites showed no potential for tissue cyst [bradyzoites] and oocysts formation in laboratory infected rats and mice [Dubey et al. 1999; Mordue et al. 2001; Asgari et al.2013]. The faster replication of the tachyzoites leads to many pathological changes ranging from mild congestion to severe degeneration involving mainly the liver, spleen and brain [Unno et al. 2013; FuentesCastro et al. 2017].

Although many drugs are used for toxoplasmosis treatment, yet no definitive "gold standard" treatment has been available. Some medicines have been commonly used such as pyrimethamine-sulfadiazine, trimethoprim-sulfamethoxazole, pyrimethamine-clindamycin, azithromycin and traditional Chinese medicine. They may be poorly tolerated, have severe side effects and/or have poor tissue penetration. Thus, the uncertainty of their efficacy is still not resolved [Valentini et al. 2015; Wei et al. 2015].

Spiramycin is less toxic than other drugs and it is used for the treatment of $T$. gondii infection among human population and in the prevention of vertical transmission from mother to foetus in the first trimester of pregnancy. It is absorbed efficiently after oral intake and spreads quickly to blood and tissues [Shi et al. 2005]. Despite its significant tissue penetration, it reveals poor crossing of the blood brain barrier [BBB] owing to the presence of the efflux transporters for which spiramycin is a substrate [Grover and Benet, 2009]. For enhancement of its brain bypass, spiramycin could be co-administered with metronidazole to inactivate the efflux pumps present in the BBB [Chew et al. 2012]. 
In the search for new strategies that overcome the disadvantage of the used treatment regimens, it was found that application of nanomedicine improved the drug biodistribution, modified bioavailability and decreased toxicity[Assolini et al. 2017].Numerous types of nanoparticles [NPs] such as silver and gold are used, but their use may be hampered by their toxic side effects, adding to their relatively higher production cost in comparison to the natural based NPs [Cameron et al. 2016; Benelli, 2018].Chitosan[CS] is a normal non-toxic polysaccharide obtained from shells of crustaceans and molluscs by chitin deacetylation [Yong et al.2015]. The advantages of CS make it the material of choice for NPs preparation in various fields. Its biocompatible, biodegradable, nontoxic nature and relatively low cost encourage its suitable applicability especially in developing countries. CS has been extensively known as an antimicrobial agent due to its intrinsic positive charge density and chelating capacity [Kong et al. 2010; Nehra et al. 2018; Ahmed et al. 2019]. Many studies have proven the therapeutic effectiveness of CS NPs against many protozoa, besides their use as vehicles to deliver anti-parasitic drugs to improve their efficacy. It is successfully used as anti-Cryptosporidium, anti-Trypanosoma, anti-Leishmania, antiPlasmodium and anti-Toxoplasma in vivo and in vitro studies [Tripathy et al. 2012; Chaubey and Mishra, 2014; Unciti-Broceta et al. 2015; Teimouri et al. 2018; Ahmed et al. 2019]. Few studies have addressed the tissue injury caused by the parasite before and after treatment [Fuentes-Castro et al. 2017].

The present study is a continuation of our previous work that was conducted by Hagras et al. [2019], who reported that spiramycin-CS NPs formulation revealed the highest therapeutic outcome in acute experimental toxoplasmosis treatment compared to CS NPs, spiramycin and spiramycin co-administered with metronidazole. This study was designed to investigate the effect of these drugs on the parasite burden and pathological changes in the liver, spleen and brain in T. gondii [RH strain] infected Swiss albino mice.

\section{Materials And Methods}

Details of the methodology are presented in the work of Hagras et al. [2019].

\section{Parasite}

Briefly, the RH virulent strain of $T$. gondii was acquired from the Parasitology Department, Faculty of Medicine, Alexandria University, Egypt. The strain was conserved by successive intraperitoneal passage of tachyzoites in Swiss albino mice. The peritoneal fluid was obtained from sacrificed mice on the 4th day post-infection and washed three times with saline. A drop of the peritoneal exudates was added to the haemocytometer and the tachyzoites number was adjusted for mice infection.

\section{Drugs}

Blank chitosan nanoparticles [CS NPs], spiramycin, spiramycin-metronidazole and spiramycin-CS NPS formulation were administered from day one of infection for 7 days. The dose per mouse was adjusted and dissolved in $100 \mu \mathrm{l}$ saline for oral administration. 


\section{II.1. Preparation of both spiramycin and spiramycin- metronidazole suspensions from tablets:}

Spiramycin tablets and metronidazole tablets [Rovamycin ${ }^{\circledR}$ and Flagy $\left.\right|^{\circledR}$-Sanofi Aventis Pharmaceutical Company, France] were purchased. Each tablet was weighed then crushed into powder. The active ingredient of each tablet was calculated according to its weight.

\section{II.2. Preparation of CS NPs and spiramycin-loaded CS NPs:}

Nanoparticles [NPs] were prepared and characterized by ionotropic gelation technique based on the interaction between the positive charge of amino groups of chitosan [CS] and the negative groups of sodium tripolyphosphate (TPP) [Hagras et al.2019].

\section{Mice:}

Seventy male Swiss Albino mice with a weight range of 20-25 grams were obtained from Medical Technology Center, Medical Research Institute, Alexandria University, Egypt. The study was approved by the ethics committee of the Medical Research Institute, based on international regulations of animal care.

\section{Experimental design [Figure 1]:}

The mice were distributed into seven experimental groups (ten mice per each). T. gondii RH strain infection was induced by intraperitoneal mice injection with 2500 tachyzoites/100 $\mu \mathrm{l}$ saline/ mouse except the healthy controls [Araujo et al.1992; Ezz Eldin et al. 2015].

The groups were divided as follows:

- Group I: Healthy control.

- Group II: Infected untreated control, each mouse received $100 \mu \mathrm{l}$ saline [the vehicle of the used drugs] orally.

- Group III: Infected mice received blank CS NPs at a dose of $30 \mathrm{mg} / \mathrm{kg}$ [Hagras et al. 2019].

- Group IV: Infected mice treated with spiramycin [Rovamycin $\left.{ }^{\circledR}\right]$ at a dose of $400 \mathrm{mg} / \mathrm{kg} / \mathrm{day}$ [Chew et al. 2012].

- Group V: Infected $\mathrm{m}$ ice received a combination of metronidazole at a dose of $500 \mathrm{mg} / \mathrm{kg}$ followed by $400 \mathrm{mg} / \mathrm{kg}$ spiramycin after 30 minutes [Chew et al., 2012].

- Group Vl: Infected mice treated with spiramycin-CS NPs formulation at a dose of $400 \mathrm{mg} / \mathrm{kg} / \mathrm{day}$.

- Group VII: Infected mice treated with spiramycin-CS NPs at a dose of $100 \mathrm{mg} / \mathrm{kg} / \mathrm{day}$.

The mice were anaesthetized and sacrificed by cervical dislocation on the 8th day i.e., 24 hours after the seven days of treatment. 


\section{Determination of parasitic burden}

Tachyzoites were counted in Giemsa-stained impression smears of liver, spleen and brain. The mean count was obtained from thirty oil immersion fields in each organ per each mouse [ten fields/ each slide and three slides/ each organ] [Eissa et al. 2012; Gaafar et al. 2014].

\section{Histopathological study}

Specimens from the liver, spleen and brain were taken from all the groups and fixed in $10 \%$ formalin. They were dehydrated in an ascending series of ethyl alcohol [70\%, 80\%, 95\% and $100 \%]$ then cleared in xylene. The samples were embedded in molten paraffin at $60^{\circ} \mathrm{C}$ for $1-2 \mathrm{hrs}$ to form paraffin blocks. Block sections were cut using microtome. Each section was $4 \mu \mathrm{m}$ thick. Two sections were prepared from the different organs of each mouse, they were mounted on slides, then left in the oven at $40^{\circ} \mathrm{C}$ to dry and fix on the slides. Slides were deparaffinized by dipping them in xylene followed by passing them in descending series of ethyl alcohol $[100 \%, 95 \%, 80 \%$ and $70 \%]$ then rinsed with water for rehydration. Slides were stained with haematoxylin, rinsed under running water then counterstained with eosin. They were dehydrated using an ascending series of ethyl alcohol and then mounted in Canada balsam. Tissue sections of the liver, spleen and brain were examined under the microscope [Titford, 2006; Musumeci, 2014]

\section{Statistical analysis}

Differences were studied by Kruskal Wallis test using IBM SPSS software package version 20.0 [Armonk, NY: IBM Corp]. They were considered significant when $\mathrm{p}<0.05$ [Kotz et al. 2006; Kirkpatrick and Feeney, 2013].

\section{Results}

\section{Parasite burden:}

The infected untreated control group showed median parasite counts of 12, 8 and 3 / 30 oil immersion field (OIF) in liver, spleen and brain respectively. There was a significant reduction in parasite burden in the groups treated with spiramycin-CS NPs formulation in comparison to other groups [Figures 1-3].

\section{Histopathological study:}

\section{II.1. Liver (Fig. 4)}

Microscopic examination of sections of the liver from healthy control (group I) showed normal hepatic architecture divided into lobules. The hepatocellular cords were mostly one or two layers thick radiating from the central vein towards the periphery of the lobule. The normal hepatocytes were polygonal in shape with centrally placed round or oval dark violet nuclei and granulated eosinophilic cytoplasm. The 
cords of hepatocytes were separated by blood sinusoids lined by endothelial cells (EC). At the periphery of the lobule, the portal triads showed no inflammatory cellular infiltrates [Figure 4.1].

In the infected untreated control (group II), microscopic examination of liver tissue revealed invasive tachyzoites aggregated among the hepatocytes. Markedly dilated and congested central veins and sinusoids with evident focal necrosis and inflammation. (Fig. 4.2)

The liver sections of CS NPs treated group (III) showed pseudocysts with necrotic tachyzoites. Residual apoptotic bodies of hepatic nuclei and degenerative hepatocytes with marked dilated congested central veins and sinusoids were noticed. Moreover, there was evidence of lobular inflammation [Figure 4.3].

In the treated spiramycin group (IV), microscopic examination of the liver demonstrated that the hepatocytes were large in size with large vesicularnuclei and vacuolar degeneration of cytoplasm. Marked dilated congested central veins and dilated sinusoids surrounded by necrotic hepatocytes with obvious inflammation [Figure 4.4].

The microscopic examination of spiramycin-metronidazole treated group (V), illustrated marked dilated sinusoids surrounded by moderate inflammatory infiltrate with interface hepatitis and moderate portal fibrosis. Most hepatocytes appeared intact but some of them were necrotic [Figure 4.5].

Spiramycin- CS NPs $400 \mathrm{mg} / \mathrm{kg}$ treated group (VI) revealed dilated sinusoids and absent inflammatory infiltrate [Figure 4.6].

The liver of spiramycin-CS NPs $100 \mathrm{mg} / \mathrm{kg}$ treated group (VII) demonstrated pseudocysts with necrotic tachyzoites. There was evidence of regenerative hepatocytes. Dilatation of central veins and sinusoids were observed with minimal inflammation formed of few perivascular macrophages [Figure 4.7].

\section{II.2. Spleen (Fig. 5)}

Microscopic examination of the spleen of the healthy control (group I) revealed well-organized white pulp (W) (containing lymphocytes) and red pulp (R) differentiated by trabecular muscles (TR) [Figure 5.1].

Infected untreated control (group II) spleen section showed pseudocysts containing tachyzoites. Marked disorganized architecture and extramedullary hematopoiesis with areas of necrosis were observed. There was an obvious decrease in lymphocyte counts and increase in histiocytes [Figures5.2 and 5.3].

In CS NPs treated group (III), areas of macrophages loaded by tachyzoites were observed. Disorganized splenic architecture in addition to extramedullary hematopoiesis were evident in the red pulp [Figure 5.4].

The microscopic examination of spiramycin infected treated group (IV) illustrated pseudocysts containing necrotic tachyzoites and numerous apoptotic cells. Severe disorganized splenic architecture with enhancement of the inflammatory cells and reduction in the lymphocytes were observed [Figure 5.5]. 
The spleen of spiramycin-metronidazole treated group $(\mathrm{V})$ demonstrated moderate disorganized architecture with extramedullary hematopoiesis and excess eosinophils [Figure 5.6].

In spiramycin-CS NPs $400 \mathrm{mg} / \mathrm{kg}$ treated group (VI), necrotic tachyzoites in pseudocysts in the red pulp and at the margin of regenerative lymphocytes of the white pulp were observed. Mild disorganized spleen architecture with evident tissue recovery and extramedullary hematopoiesis were noticed [Figure 5.7].

Spiramycin CS NPs $100 \mathrm{mg} / \mathrm{kg}$ treated group (VII) showed pseudocysts with necrotic tachyzoites. Moderate disorganized spleen architecture with regenerative white pulp forming well defined germinal centres as well as frequent epithelioid histiocytes were evident [Figure 5.8].

\section{II.3. Brain (Fig. 6)}

Examination of the brain cortex of the healthy control [group I] revealed normal architecture composed of neuroglial cells with ill-defined cytoplasm and pyramidal cells. Normal blood vessels were present [Figure 6.1].

In infected untreated control (group II), the cerebral cortex showed gliosis, apoptosis as well as perivascular and parenchymal inflammation [Figure 6.2].

The microscopic examination of CS NPs treated group (III) illustrated mild dilated blood vessels. Small pyramidal cells and others with vacuolated cytoplasm were noticed. Apoptosis and marked gliosis were observed [Figure 6.3].

In the spiramycin treated group (IV), the brain revealed the occurrence of perivascular inflammatory infiltrate, gliotic nodules and necrotic neurons [Figure 6.4].

In the brain of spiramycin-metronidazole treated group (V), no tachyzoites were detected. Mild congested blood vessels, moderate gliosis and ferrugination (mineralized bodies of necrotic neurons) were observed [Figure 6.5].

Spiramycin- CS NPs $400 \mathrm{mg} / \mathrm{kg}$ treated group (VI) revealed absence of tachyzoites with mild dilation of blood vessels. Normal neuroglial cells with no evidence of inflammation were observed [Figure 6.6].

In the brain of spiramycin-loaded CS NPs $100 \mathrm{mg} / \mathrm{kg}$ treated group (VII) spongiosis, moderate gliosis and apoptosis were seen. No inflammation was observed [Figure 6.7].

\section{Discussion}

Toxoplasmosis treatment is a worthy challenge to achieve, particularly that the parasite is intracellular and crosses the blood brain barrier [Briones et al. 2008].

To fulfil the present work, CS NPs, spiramycin, spiramycin co-administered with metronidazole and spiramycin-CS NPs were evaluated for treating acute toxoplasmosis regarding parasite load and 
histopathology of the liver, spleen and brain in Swiss albino mice.

The parasite burden decreased in liver, spleen and brain among all treated mice in comparison to the infected untreated control. The least parasite load was obtained after the treatment with spiramycinloaded NPs compared to the other groups regarding all the studied organs.

In the present work, histopathological study demonstrated severe tissue damage in the liver, spleen and brain of acute T. gondii infected untreated mice. Marked inflammation, congestion, areas of lytic necrosis and free tachyzoites within tissue sections were detected as compared to those of the healthy control and of the treated mice. Activated inflammatory cells that were attracted by the free tachyzoites, triggered the inflammatory response, causing cell lysis. Their effect incorporates with the mechanical damage produced by the tachyzoites. These findings coincide with various reports of previous studies [Unno et al. 2013; Fuentes-Castroet al. 2017].

All the used treatments [CS NPs, spiramycin, spiramycin co-administered with metronidazole and spiramycin-CS NPs] modulated the pathological effects in the liver, spleen and brain of mice infected with T. gondii RH strain. The various degrees of response were characterized by disappearance of necrosis and reduction of inflammation.

Concerning the liver of infected untreated mice, it was characterized by congested veins and sinusoids accompanied by lobular necrosis, vacuolar degeneration of hepatocytes and inflammatory infiltrate in both lobular and portal areas with interphase hepatitis. Free tachyzoites were seen in the necrotic foci. The findings agreed with those described by Unno et al.[2013] and Mady et al.[2016]. All treated groups showed reduction in the inflammatory infiltrate with fibrosis. Regeneration of hepatocytes was noted together with minimal mononuclear inflammatory infiltrate. In mice receiving spiramycin-metronidazole, residual portal and bridging fibrosis were noted. Interestingly, the groups of spiramycin-CS NPs 400 $\mathrm{mg} / \mathrm{kg}$ treated and spiramycin-CS NPs $100 \mathrm{mg} / \mathrm{kg}$ showed nearly normal architecture with necrotic tachyzoites.

Regarding the spleen of the infected untreated group, there was disorganized architecture with areas of necrosis and absent germinal centers. Extramedullary hematopoiesis appeared with frequent megakaryocytes. This latter sign was previously described in the literature and was clarified by the fact that $T$. gondii induces systemic levels of IL-12 which promotes high level of extramedullary hematopoiesis [EMH] [Zaretsky et al. 2012]. In the spleen of the treated groups, extramedullary hematopoiesis persisted. However, in the groups treated with spiramycin-CS NPs $400 \mathrm{mg} / \mathrm{kg}$ and spiramycin-loaded CS NPs $100 \mathrm{mg} / \mathrm{kg}$, the necrosis disappeared completely and restoration of close normal architecture was identified. There was regeneration of white pulp, well-structured lymphoid follicles with reactive germinal centers and healthy demarcated red pulp.

In the brain of the infected untreated mice, the injury was evidenced by astrocytic proliferation [gliosis] and scattered necrotic neurons. Perivascular inflammation, spongiosis and congestion were observed. Similar observations were reported by Parlog et al. [2015] and Fuentes-Castro et al. [2017]. Treatment 
greatly reduced the inflammation; dead neurons were mineralized or replaced by gliotic nodules. Best results were seen in the group treated with spiramycin-CS NPs $400 \mathrm{mg} / \mathrm{kg}$; normal neuroglial cells with no sign of inflammation were detected.

Conclusion: The most significant tissue regenerative effect in liver, spleen and brain with absence of living tachyzoites was noticed in the mice treated with spiramycin-CS NPs $400 \mathrm{mg} / \mathrm{kg}$. Thus, it could be a promising treatment for toxoplasmosis due to its capability to penetrate the tissues and particularly the BBB.

\section{Declarations}

\section{Conflict of interest}

The authors declare that there is no conflict of interest regarding the publication of this article.

\section{Acknowledgment}

We gratefully acknowledge Unipharm Pharmaceutical Company, Egypt for kindly providing spiramycin powder used in the nanoparticle's preparation.

\section{References}

1. Ahmed, SA, El-Mahallawy, HS and Karanis, $P$ [2019] Inhibitory activity of chitosan nanoparticles against Cryptosporidium parvum oocysts. Parasitology Research 118 [7], 2053-2063. doi: 10.1007/s00436-019-06364-0.

2. Araujo, FG, Prockocimer, P, Lin, L and Remington, SJ [1992] Activity of clarithromycin alone or in combination with other drugs for treatment of murine toxoplasmosis. Antimicrobial Agents and Chemotherapy 36, 2454-2457. doi: 10.1128/aac.36.11.2454.

3. Asgari, Q, Keshavarz, H, Shojaee, S, Motazedian, MH, Mohebali, M, Miri, R, Mehrabani, D and Rezaeian, M [2013] In Vitro and In Vivo Potential of RH Strain of Toxoplasma gondii [Type I] in Tissue Cyst Forming. Iran Journal of Parasitology 8[3], 367-375.

4. Assolini, JP, Concato, VM, Gonçalves, MD, Carloto, ACM, Conchon-Costa, I, Pavanelli, WR, Melanda,FN and Costa, IN [2017] Nanomedicine advances in toxoplasmosis: diagnostic, treatment, and vaccine applications. Parasitology Research 116 [6], 1603-1615. doi: 10.1007/s00436-017-54582.

5. Benelli, G [2018] Gold nanoparticles - against parasites and insect vectors. Acta Tropica 178, 73-80. doi: 10.1016/j.actatropica.2017.10.021.

6. Briones, E, Colino, Cl and Lanao, JM [2008] Delivery systems to increase the selectivity of antibiotics in phagocytic cells. Journal of Controlled Release 125 (3), 210-227. doi:

10.1016/j.jconrel.2007.10.027. 
7. Cameron, P, Gaiser, BK, Bhandari, B, Bartley, PM, Katzer, F and Bridle, H [2016] Silver nanoparticles decrease the viability of Cryptosporidium parvum oocysts. Applied and Environmental Microbiology 82, 431-437. doi: 10.1128/AEM.02806-15.

8. Chaubey, P and Mishra, B [2014] Mannose-conjugated chitosan nanoparticles loaded with rifampicin for the treatment of visceral leishmaniasis. Carbohydrate Polymers 101, 1101-1108. doi: 10.1016/j.carbpol.2013.10.044.

9. Chew, W, Segarra, I, Ambu, S and Mak, JW [2012] Significant reduction of brain cysts caused by Toxoplasma gondii after treatment with spiramycin coadministered with metronidazole in a mouse model of chronic toxoplasmosis. Antimicrobial Agents and Chemotherapy 56, 1762-1768. doi: 10.1128/AAC.05183-11.

10. Dubey, JP, Shen, SK, Kwok, OC and Frenkel, JK [1999] Infection and immunity with the RH strain of Toxoplasma gondii in rats and mice. The Journal of Parasitology 85, 657-662.

11. Eissa, MM, El-Azzouni, MZ, Mady, RF, Fathy, FM and Baddour, NM [2012] Initial characterization of an autoclaved Toxoplasma vaccine in mice. Experimental Parasitology 131 (3), 310-316. doi: 10.1016/j. Exp Para.2012.05.001.

12. Ezz Eldin, HM, Kamel, HH, Badawy, AF and Shash, LS [2015] A comparative study between excretory/secretory and autoclaved vaccines against $\mathrm{RH}$ strain of Toxoplasma gondii in murine models. Journal of Parasitic Diseases 39(3), 526-535. doi:10.1007/s12639-013-0390-6.

13. Fuentes-Castro, BE, Reyes-García, JG, Valenzuela-Vargas, MT and Martínez-Gómez, F [2017] Histopathology of murine toxoplasmosis under treatment with dialyzable leukocyte extract. Memorias do Instituto Oswaldo Cruz 112 [11], 741-747. doi: 10.1590/0074-02760170045.

14. Gaafar, MR, Mady, RF, Diab, RG and Shalaby, TI [2014] Chitosan and silver nanoparticles: promising anti-Toxoplasma agents. Experimental Parasitology 143, 30-38. doi: 10.1016/j.exppara.2014.05.005.

15. Grover, A and Benet, LZ [2009] Effects of drug transporters on volume of distribution. The AAPS Journal 11, 250-261. doi: 10.1208/s12248-009-9102-7.

16. Hagras, NA, Allam, AF, Farag, HF, Osman, MM, Shalaby, TI, Mogahed, NMFH, Tolba MM and Shehab AY (2019) Successful treatment of acute experimental toxoplasmosis by spiramycin-loaded chitosan nanoparticles. Experimental Parasitology 204, 107717. doi: 10.1016/j.exppara.2019.107717.

17. Kirkpatrick, LA and Feeney, BC [2013] A Simple Guide to IBM SPSS Statistics for Version20.0. Cengage Learning, California.

18. Kong, $M$, Chen, $X G$, Xing, $K$ and Park, HJ [2010] Antimicrobial properties of chitosan and mode of action: a state of the art review. International Journal of Food Microbiology 144, 51-63. doi: 10.1016/j.jfoodmicro.2010.09.012.

19. Kotz, S, Balakrishnan, N, Read, CB, Vidakovic, B and Johnson, NL [2006] Encyclopaedia of Statistical Sciences, 2nd Edn. USA: Wiley-Interscience.

20. Mady, RF, El-Hadidy, W and Elachy, S [2016] Effect of Nigella sativa oil on experimental toxoplasmosis. Parasitology Research 115[1], 379-390. doi: 10.1007/s00436-015-4759-6. 
21. Mordue DG, Monroy F, La Regina M, Dinarello CA and Sibley LD [2001] Acute toxoplasmosis leads to lethal overproduction of Th1 cytokines. Journal of Immunology 167, 4574-4584. doi: 10.4049/jimmunol.167.8.4574.

22. Musumeci, G [2014] Past, present and future: overview on histology and histopathology. Journal of Histology and Histopathology 1, 5. doi: 10.7243/2055-091X-1-5.

23. Nehra, $P$, Chauhan, R, Garg, N and Verma, K [2018] Antibacterial and antifungal activity of chitosan coated iron oxide nanoparticles. British Journal of Biomedical Science 75, 13-18. doi: 10.1080/09674845.2017.1347362.

24. Parlog, A, Schlüte, D and Dunay, IR [2015] Toxoplasma gondii-induced neuronal alterations. Parasite Immunology 37(3), 159-170. doi: 10.1111/pim.12157.

25. Shi, XG, Fawcett, JP, Chen, XY and Zhong, DF [2005] Structural identification of bitespiramycin metabolites in rat: a single oral dose study. Xenobiotica 35, 343-358. doi: 10.1080/00498250500087580.

26. Teimouri, A, Azami, SJ, Keshavarz, H, Esmaeili, F, Alimi, R, Mavi, SA and Shojaee, S [2018] AntiToxoplasma activity of various molecular weights and concentrations of chitosan nanoparticles on tachyzoites of RH strain. International Journal of Nanomedicine 13, 1341-1351. doi: 10.2147/IJN.S158736.

27. Titford, M [2006] A Short History of Histopathology Technique. Journal of Histotechnology 29, 99110. doi: 10.1179/his.2006.29.2.99.

28. Tripathy, S, Das, S, Chakraborty, SP, Sahu, SK, Pramanik, P and Roy, S [2012] Synthesis, characterization of chitosan-tripolyphosphate conjugated chloroquine nanoparticle and its in vivo anti-malarial efficacy against rodent parasite: a dose and duration dependent approach. International Journal of Pharmaceutics 434, 292-305. doi: 10.1016/j.ijpharm.2012.05.064.

29. Unciti-Broceta, JD, Arias, JL, Maceira, J, Soriano, M, Ortiz-González, M, Hernández-Quero, J, MuñózTorres, M, de Koning, HP, Magez, S and Garcia-Salcedo, JA[2015] Specific cell targeting therapy bypasses drug resistance mechanisms in African trypanosomiasis. PLoS Pathogens 11, e1004942. doi: 10.1371/journal.ppat.1004942.

30. Unno, A, Kachi , S, Batanova, TA, Ohno, T, Elhawary, N, Kitoh. K and Takashima, Y [2013] Toxoplasma gondii tachyzoite- infected peripheral blood mononuclear cells are enriched in mouse lungs and liver. Experimental Parasitology 134(2), 160-164. doi: 10.1016/j.exppara.2013.03.006.

31. Valentini, P, Buonsenso, D, Barone, G, Serranti, D, Calzedda, R, Ceccarelli, M,Speziale, D, Ricci,R and Masini,L[2015] Spiramycin/cotrimoxazole versus pyrimethamine/sulfonamide and spiramycin alone for the treatment of toxoplasmosis in pregnancy. Journal of Perinatology 35[2], 90-94. doi: 10.1038/jp.2014.161.

32. Wei, HX, Wei, SS, Lindsay, DS and Peng, HJ [2015] A systematic review and meta-analysis of the efficacy of anti-Toxoplasma gondii medicines in humans. PLoS One 10 [9], e0138204. doi: $10.1371 /$ journal.pone.0138204. 
33. Wohlfert, EA, Blader, IJ and Wilson, EH [2017] Brains and brawn: toxoplasma infections of the central nervous system and skeletal muscle. Trends in Parasitology 33[7], 519-531. doi: 10.1016/j.pt.2017.04.001.

34. Yong, SK, Shrivastava, M, Srivastava, P, Kunhikrishnan, A and Bolan, N [2015] Environmental applications of chitosan and its derivatives. Reviews of Environmental Contamination and Toxicology 233, 1-43. doi: 10.1007/978-3-319-10479-9_1.

35. Zaretsky, AG, Silver, JS, Siwicki, M, Durham, A, Ware, CF and Hunter, CA [2012] Infection with Toxoplasma gondii alters lymphotoxin expression associated with changes in splenic architecture. Infection and Immunology 80 [10], 3602-3610. doi: 10.1128/IAI.00333-12.

\section{Figures}

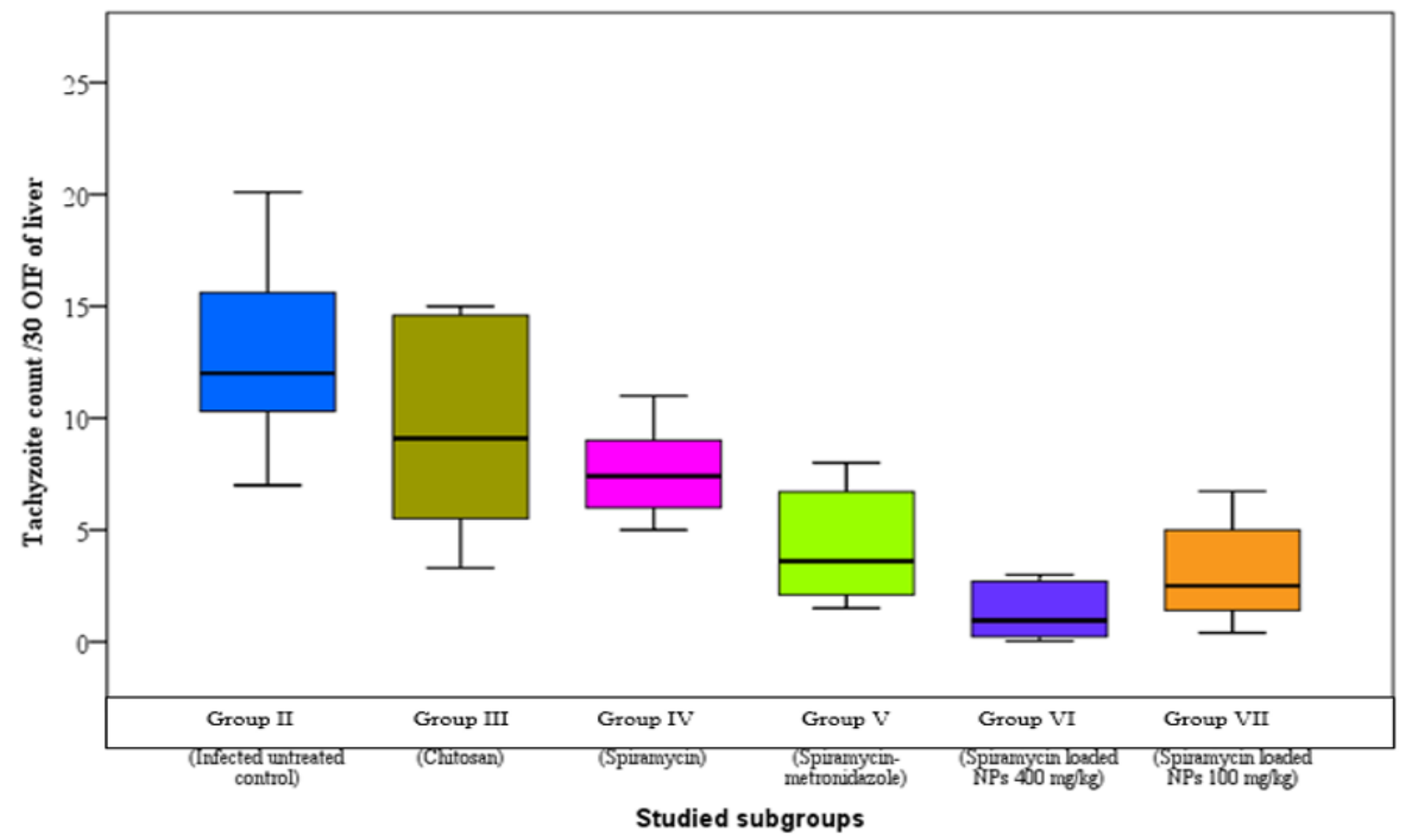

\section{Figure 1}

Tachyzoites count in liver impression smears of the different studied groups. 


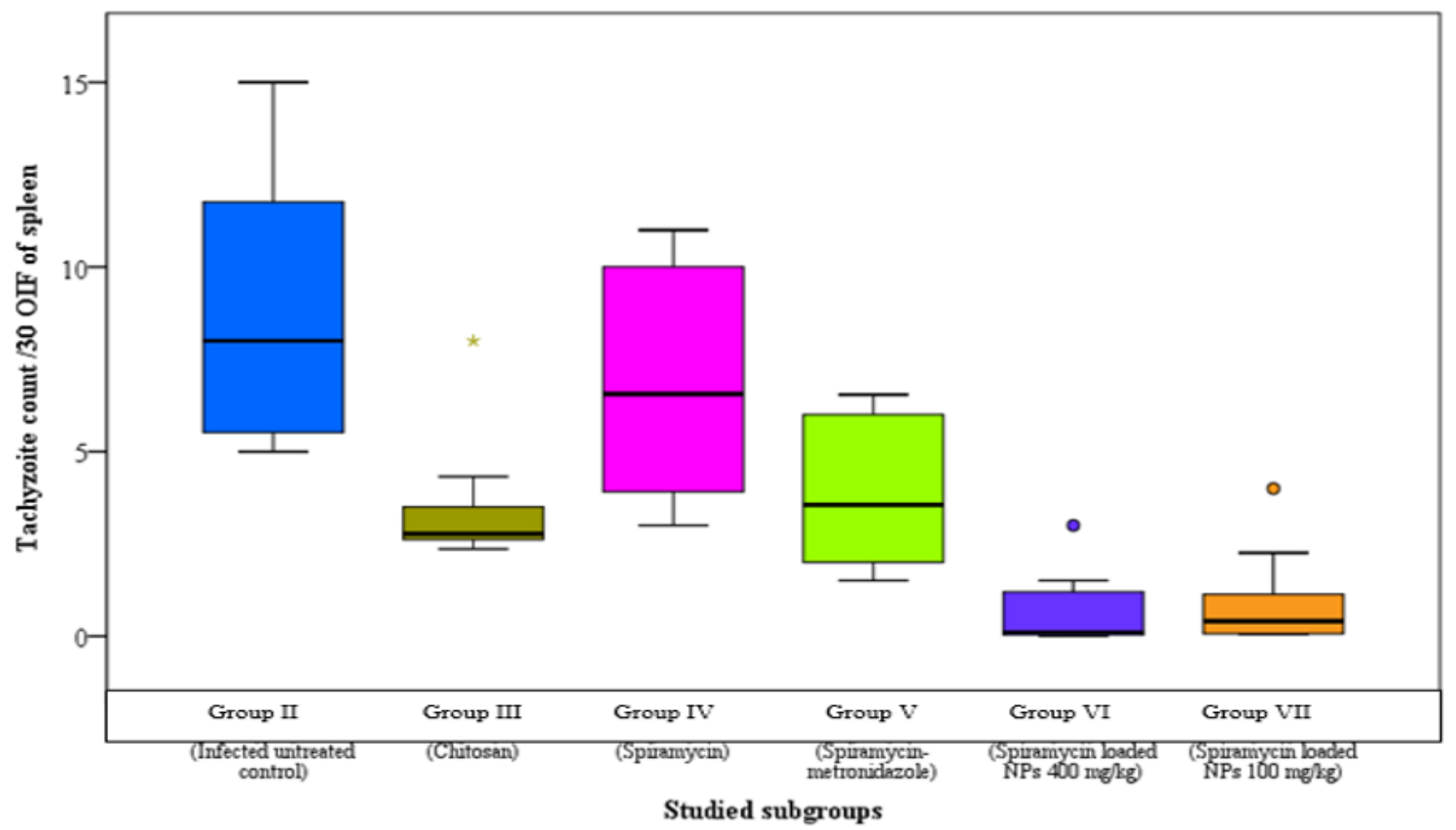

Figure 2

Tachyzoites count in spleen impression smears of the different studied groups. 


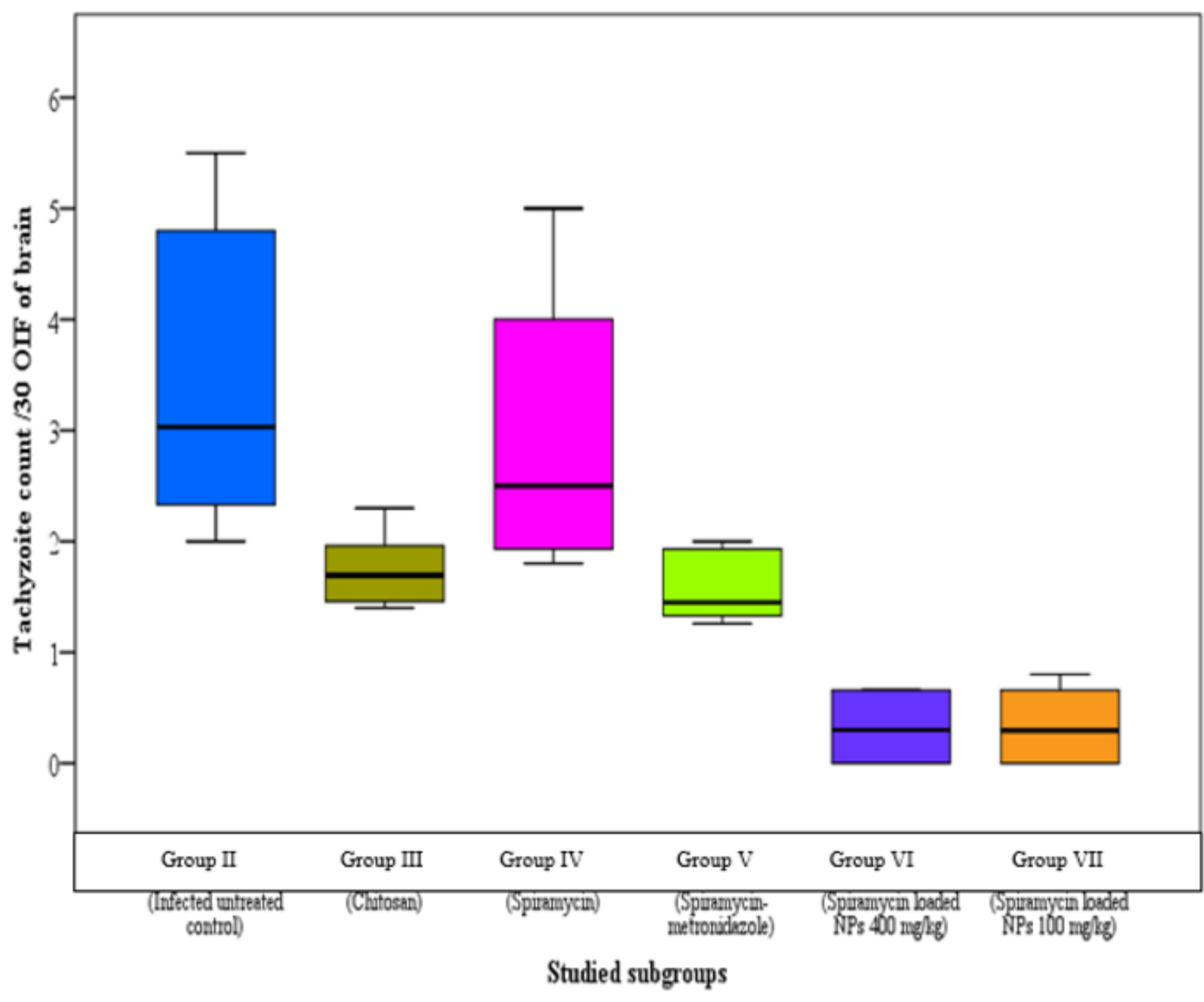

Figure 3

Tachyzoites count in brain impression smears of the different studied groups. 


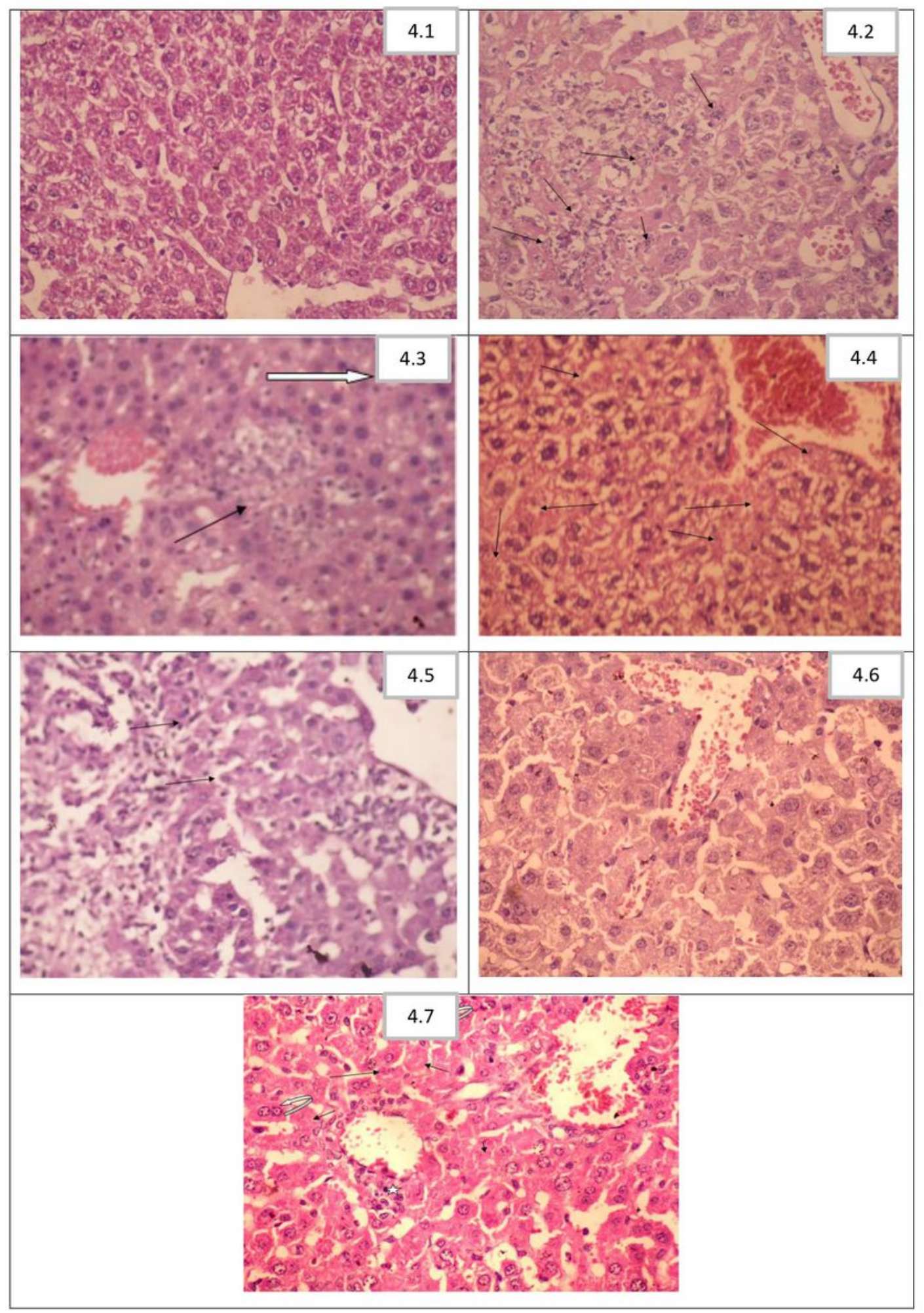

\section{Figure 4}

Histopathological study of the liver. Figure 4.1: Liver section of healthy control (group I) showing normal architecture. [H\&E X400] Figure 4.2: Liver section of infected untreated control (group II) showing focal necrosis with acute and chronic mononuclear inflammatory infiltrate. Tachyzoites are seen among the hepatocytes [arrows]. Marked dilated congested central vein and the sinusoids are observed. [H\&E X400] Figure 4.3: Liver section of CS NPs treated group (III) showing marked dilated congested central vein and 
sinusoids. Foci of hepatocellular necrosis and inflammatory cell infiltration(arrow). Pseudocysts with necrotic tachyzoites are observed [white arrow]. [H\&E X400] Figure 4.4: Liver section of spiramycin treated group (IV) showing markedly dilated congested sinusoid. Dispersed necrotic hepatocytes [arrows] and widespread vacuolar degeneration. [H\&E X400] Figure 4.5: Liver section of spiramycin-metronidazole treated group $(\mathrm{V})$ showing dilated sinuses, moderate portal inflammatory infiltrate with fibrosis and interface hepatitis [arrows]. [H\&E X400] Figure 4.6: Liver section of spiramycin-CS NPs $400 \mathrm{mg} / \mathrm{kg}$ treated group ( $\mathrm{VI})$ showing dilated congested sinusoid with near normal architecture. [H\&E X400] Figure 4.7: Liver section of spiramycin-CS NPs $100 \mathrm{mg} / \mathrm{kg}$ treated group (VII) showing dilatation and congestion of portal and central veins. Minimal perivascular inflammatory infiltrate formed mainly of macrophages [star]. Pseudocysts with necrotic tachyzoites are identified (arrows) as well as binucleated regenerating hepatocytes [curved arrows]. [H\&E X400] 


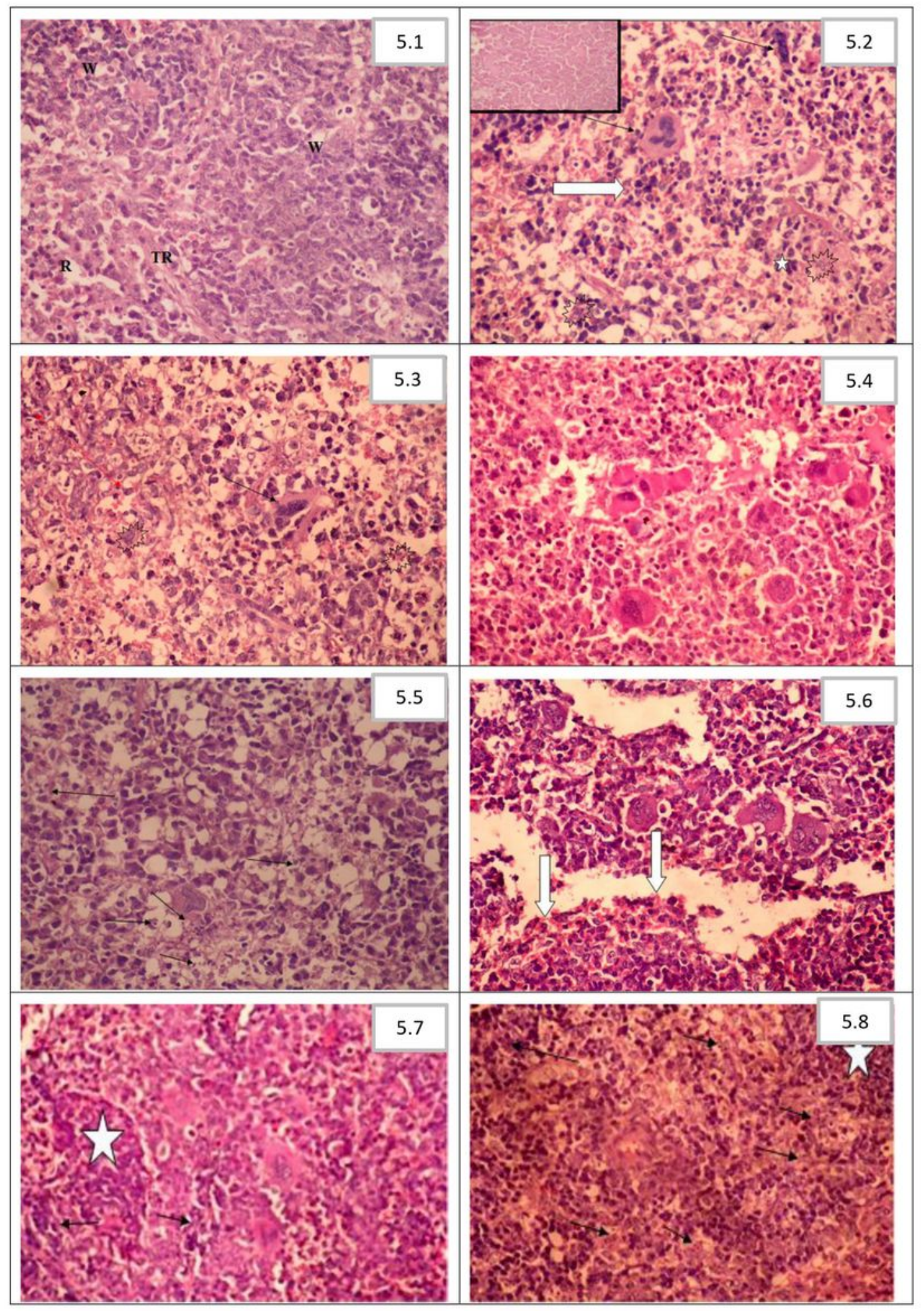

\section{Figure 5}

Histopathological study of the spleen. Figure 5.1: Spleen section of healthy control (group I) showing normal architecture. [H\&E X400] Figures 5.2 \& 5.3: Spleen section of infected untreated control (group II) showing tachyzoites [explosion shapes], marked disorganized splenic architecture with areas of necrosis [inset and red arrow] and presence of extramedullary hematopoiesis [megakaryocytes: black arrows, normoblasts: white arrow and myeloid cells: star]. [H\&E X400] Figure 5.4: Spleen section of CS NPs 
treated group (III) showing disorganized architecture with extramedullary hematopoiesis. [(H\&E X400] Figure 5.5: Spleen section of spiramycin treated group (IV) showing severe disorganized architecture with necrotic tachyzoites [arrows] and apoptotic cells. [H\&E X400] Figure 5.6: Spleen section of spiramycinmetronidazole treated group $(\mathrm{V})$ showing moderate disorganized architecture with extramedullary hematopoiesis and excess eosinophils [white arrows]. [H\&E X400] Figure 5.7: Spleen section of spiramycin-CS NPs $400 \mathrm{mg} / \mathrm{kg}$ treated group (VI) showing mild disorganized architecture with extramedullary hematopoiesis and frequent necrotic tachyzoites [arrows]. Regenerating white pulp is evident [star]. [H\&E X400] Figure 5.8: Spleen section of spiramycin-CS NPs $100 \mathrm{mg} / \mathrm{kg}$ treated group (VII) showing moderate disorganized spleen architecture with frequent epithelioid histiocytes loaded with necrotic tachyzoites [arrows]. Regenerating white pulp is noticed [star]. [H\&E X400] 


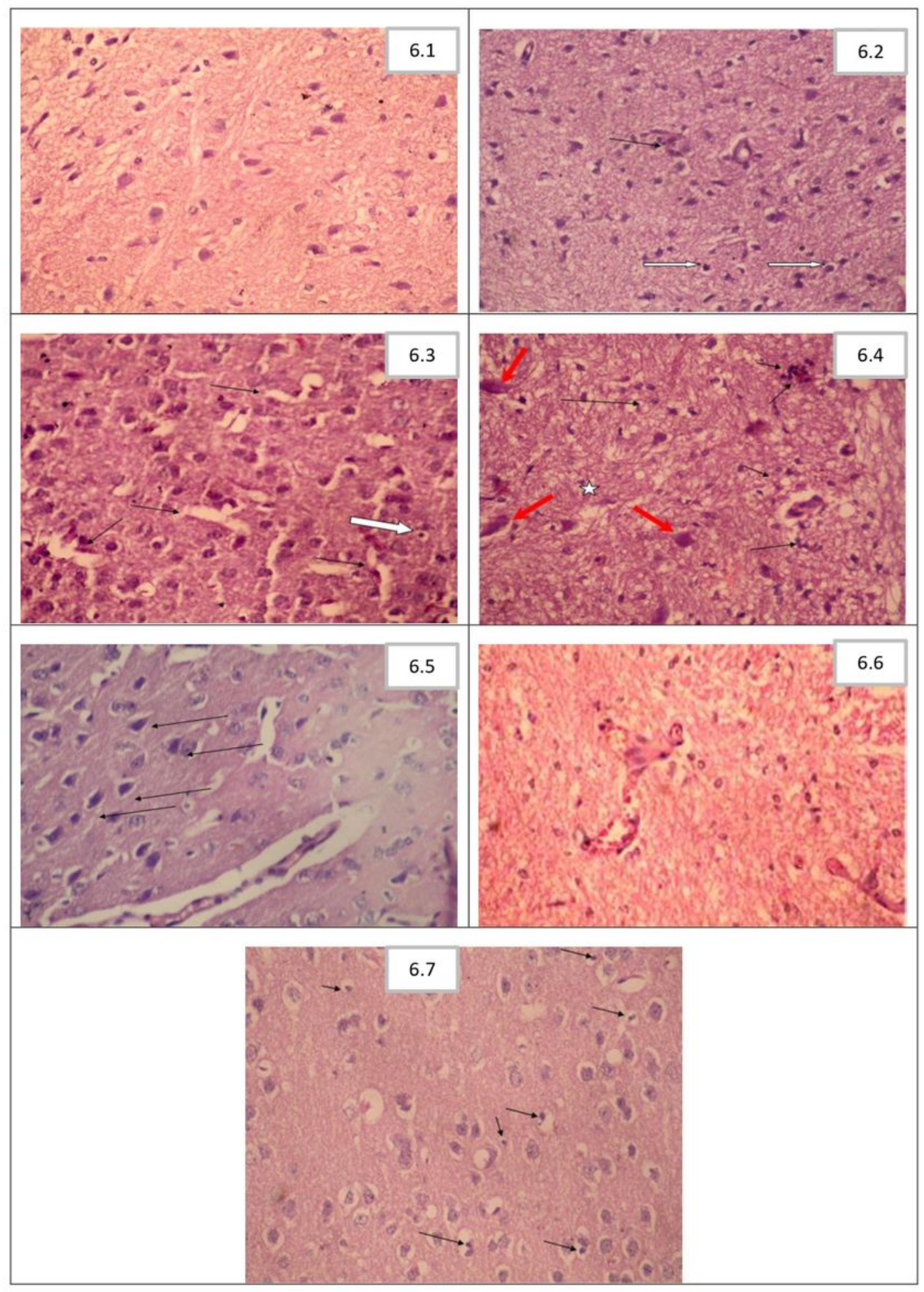

\section{Figure 6}

Histopathological study of the brain. Figure 6.1: Section in brain of healthy control (group I) showing normal cortex architecture. [H\&E X400] Figure 6.2: Section of cerebral cortex of infected untreated control (group II) showing gliosis, apoptosis [white arrows] and perivascular inflammatory infiltrate [arrow]. [H\&E X400] Figure 6.3: Brain section of CS NPs treated group (III) showing astrocyte proliferation [gliosis], apoptosis [white arrow] and dilated sinuses [arrows]. [H\&E X40] Figure 6.4: Brain section of spiramycin 
treated group (IV) showing gliotic nodule [star], dead neurons [red arrows] together with perivascular and parenchymal inflammatory infiltrate [black arrows]. [H\&E X400] Figure 6.5: Brain section of spiramycinmetronidazole treated group $(\mathrm{V})$ showing mild congested blood vessels, moderate gliosis and mineralized bodies of necrotic neurons so called ferrugination [arrows]. [H\&E X400] Figure 6.6: Brain section of spiramycin-CS NPs $400 \mathrm{mg} / \mathrm{kg}$ treated group (VI) showing mild dilation of blood vessels (BV) with absence of tachyzoites and near normal architecture. [H\&E X 400] Figure 6.7: Brain section of spiramycinCS NPs $100 \mathrm{mg} / \mathrm{kg}$ treated group (VII) showing moderate gliosis, dispersed apoptotic cells [arrows] and extensive vacuolation in the cortex [spongiosis]. [H\&E X 400] 